\title{
Malaria and Anaemia in Pregnant and Non-Pregnant Women of Child-Bearing Age at the University Hospital, Kumasi, Ghana*
}

\author{
Samuel C. K. Tay ${ }^{1}$, Eric Agboli ${ }^{1}$, Harry Hoffman Abruquah ${ }^{2}$, Williams Walana ${ }^{3 \#}$ \\ ${ }^{1}$ Department of Clinical Microbiology, School of Medical Sciences, Kwame Nkrumah University of \\ Science and Technology (KNUST), Kumasi, Ghana \\ ${ }^{2}$ University Hospital, Division of Microbiology and Infectious Diseases, Kwame Nkrumah University of \\ Science and Technology (KNUST), Kumasi, Ghana \\ ${ }^{3}$ Department of Clinical Laboratory Sciences, School of Medicine and Health Sciences (SMHS), \\ University for Development Studies (UDS), Tamale, Ghana \\ Email: "walanawilliams@yahoo.com
}

Received July 31, 2013; revised August 31, 2013; accepted September 7, 2013

Copyright (C) 2013 Samuel C. K. Tay et al. This is an open access article distributed under the Creative Commons Attribution License, which permits unrestricted use, distribution, and reproduction in any medium, provided the original work is properly cited.

\begin{abstract}
Background: Malaria infection during pregnancy is a major public health problem in tropical and subtropical regions globally. Anaemia is often an adverse outcome of severe parasitic infections during pregnancy in developing countries. Pregnant women in malaria-endemic communities are more susceptible to Plasmodium falciparum infections than non-pregnant women of child-bearing age. Objective: To comparatively investigate malaria and anaemia in pregnant and non-pregnant women of child-bearing age. Design: A cross-sectional comparative study. Three hundred and eighty pregnant women and 380 non-pregnant women were screened for the study. Setting: The study was conducted at the University Hospital, Kwame Nkrumah University of Science and Technology, Kumasi, Ghana. Measurements: Participants' demographic data were collected via the administration of questionnaires. In addition their blood samples were analyzed for haemoglobin level and malaria parasites, while stool samples from the pregnant women were examined for intestinal parasites. Results: The study revealed that pregnant women have higher malaria parasitaemia (12.6\%) and anaemia $(62.6 \%)$. The species of Plasmodium isolated from the pregnant women were $P$. falciparum $(85.4 \%), P$. malariae $(4.2 \%)$ and $P$. ovale $(10.4 \%)$. Malaria parasitaemia was higher in the primigravidae $(14 \%)$. However multigravidae recorded the highest anaemia prevalence $(67.1 \%)$. Age of pregnant women was a factor affecting malaria parasitaemia with a significant P-value and OR $(\mathrm{P}$ value $=0.0041,0 \mathrm{R}=7.61)$. Conclusions: Pregnant women were more susceptible to malaria and anaemia than non-pregnant women of child-bearing age. Most of the pregnant women reported at antenatal clinic during the second trimester. Primigravidae however recorded the highest malaria parasitaemia. The main species of Plasmodium observed in the blood samples was falciparum.
\end{abstract}

Keywords: Malaria; Anaemia; Pregnant Women; Non-Pregnant Women

\section{Introduction}

Malaria is a serious public health problem particularly in pregnant women in the tropics [1]. Plasmodium falciparum is responsible for the majority of malaria infections

\footnotetext{
${ }^{*}$ Competing interest: The authors declare that they have no competing interests.

Authors' contributions: Samuel C. K. Tay conceived and developed the study protocol. Eric Agboli collected and analyzed the data for the study. Harry H. Abruquah and Williams Walana participated in data analysis, and manuscript development. All authors read and approved the manuscript.

${ }^{\#}$ Corresponding author.
}

that occur in pregnancy as compared to other species of the parasite [2]. Its rate of infection in pregnancy is twice that in non-pregnant women due to physiological changes and suppressed immunity during pregnancy [3]. Without appropriate intervention, $P$. falciparum infection in pregnancy could have significant adverse consequences for both mother and foetus [4]. Malaria is more frequent and more severe in pregnant women than in age-matched controls [5]. Low endemic areas such as Southeast Asia, sporadically suffer severe or complicated malaria [4]. There is evidence that severe malaria may also be a significant problem in pregnant women in urban areas in 
sub-Saharan Africa [6].

Severe anaemia predominates as the main feature of severe malaria in areas with high levels of transmission, while hypoglycemia, respiratory failure, and cerebral malaria may predominate in areas with low levels of malaria transmission [7,8]. Anaemia is more frequent in pregnant women, and more pronounced in primigravidae than in multigravidae [9]. It is estimated that approximately $50 \%$ of pregnant women in malaria-endemic countries of Africa are anaemic [10,11]. In addition, more than half of pregnant women in the world have haemoglobin level indicative of anaemia $(<11.0$ gldl). The prevalence may however be as high as $56 \%$ or $61 \%$ in developing countries [12]. Anemia is usually multifactorial in origin and although malaria is an important contributor, nutritional deficiencies (iron and folate), other infectious diseases (hookworm, schistosomiasis and HIV) and genetic red blood cell disorders (such as sickle cell and thalassaemias) are also important contributing factors [13].

Each year, approximately 25 million African women become pregnant in malaria endemic areas [14]. The problem is quite enormous in sub-Saharan Africa where $80 \%-90 \%$ of the world's malaria cases occur. This suggests that about $19-24$ million women in the sub-region are prone to malaria and malaria-associated complication [15]. Economically, billions of dollars are lost annually through malaria [16].

Little information is currently available on the epidemiology and impact of malaria during pregnancy and in non-pregnant women of child-bearing age in the Ashanti Region of Ghana. This study focused on investigating comparatively malaria and anaemia, and the associated risk factors, among pregnant women and non-pregnant women of child-bearing age at the University Hospital, Kwame Nkrumah University of Science and Technology, Ghana.

\section{Methodology}

\subsection{Study Area and Population}

The study was conducted at the University Hospital, Kwame Nkrumah University of Science and Technology (KNUST), Kumasi, Ghana. Kumasi is the second largest city in Ghana, located in the rainforest zone of West Africa with a population of 1.5 million inhabitants of which $51 \%$ are women [17]. The climatic conditions of Kumasi are typical of that of a tropical region and therefore aid the transmission of malaria parasites. The study was carried out between February, 2010 and December, 2010. A total of 760 subjects comprising 380 pregnant women and 380 non-pregnant women of child-bearing age attending antenatal care at the hospital were enrolled.

\subsection{Blood Sample Collection and Examination}

Blood samples were collected by venipuncture. The blood samples were analyzed for haemoglobin levels, using hematology analyzer (Sysmex, KX-21N, Japan). Thick and thin films of the blood samples were giemsa stained and examined for malaria parasites using standard procedures as described by Cheesbrough [18].

\subsection{Stool Sample Collection and Examination}

Stool samples were collected from pregnant women to determine the presence of intestinal parasites. The specimens were examined microscopically using the Direct Wet Mount Technique as described by Cheesbrough [18], to detect parasite ova and larvae. Briefly, a smear was prepared from each of the fresh samples by emulsifying about $2 \mathrm{mg}$ of the stool sample on a clean $26 \times 76 \mathrm{~mm}$ glass slide in a drop of lugol's iodine. The sample was covered with a $22 \times 22 \mathrm{~mm}$ cover slip and observed using low power $(\times 10)$ and high power $(\times 40)$ objectives for the identification of protozoan trophozoites and cyst, helminth ova and larvae.

\subsection{Statistical Analyses}

Data were analyzed using Microsoft Office Excel 2007, Epi Info 6 version and GraphPad Prism 5.02 version statistical softwares. The results were presented as simple frequencies and percentages. Odds ratios were calculated with $95 \%$ confidence interval $(\mathrm{CI})$ to measure the strengths of associations between variables. P-values provide a sense of the strength of the evidence against null hypothesis.

\subsection{Ethical Consideration}

Ethical clearance for the study was obtained (Ref: CHRPE/Student/195/10) from the Ethical Review Committee of the School of Medical Sciences, Kwame Nkrumah University of Science and Technology, Kumasi. Permission to undertake the study at the University Hospital, KNUST, was granted by the hospital management and the head of the laboratory. The consent of every participant was sought.

\section{Results}

Pregnant women had $12.6 \%$ malaria prevalence while non-pregnant women of child-bearing age had $6.6 \%$. Anaemia prevalence rates in pregnant and non-pregnant women were $62.6 \%$ and $53.2 \%$ respectively (Figure 1).

The species of Plasmodium found in the pregnant women were P. falciparum (85.4\%), P. ovale $(10.4 \%)$ and $P$. malariae $(4.2 \%)$. Among the non-pregnant women of child-bearing age, $76 \%$ P. falciparum, $16 \%$ 


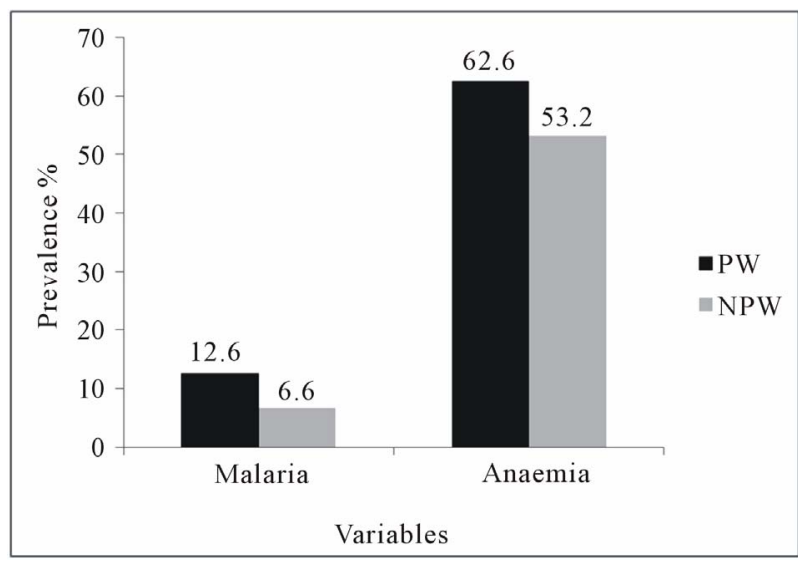

Figure 1. Comaprison of prevalence of malaria and anaemia in pregnant and non-pregnant women of child-bearing age. $\mathrm{PW}=$ Pregnant women, NPW = Non-pregnant women.

P. ovale, and $8 \%$ P. malariae were found. There was no mixed infection and no $P$. vivax identified in the study (Table 1).

Intestinal nematodes found in the stool specimens analyzed included; Ascaris lumbricoides (2), Trichuris trichiura (1), and Strongyloides stercoralis (2). Out of the 380 pregnant women, $5(1.3 \%)$ were infected with intestinal nematodes. Two $(40 \%)$ pregnant women infected with intestinal nematodes had anaemia. However, this was not significantly associated with intestinal nematodes (Table 2). Co-infection of malaria with intestinal nematodes was not recorded in the pregnant women.

The age of pregnant and non-pregnant women of child-bearing age ranged from 16 to 45 years. Young pregnant $(<20$ years) and young non-pregnant women $(<20$ years) had $5(50 \%)$ and $3(10.3 \%)$ malaria prevalence respectively. This gave an Odds Ratio (OR) of 7.61 at $95 \% \mathrm{CI}(2.1-27.4)$ in the pregnant women and an OR of 1.73 at $95 \% \mathrm{CI}(0.5-6.2)$ in the non-pregnant women of child-bearing age. Among the old women ( $\geq 20$ years), $43(11.6 \%)$ of the pregnant women and $22(6.3 \%)$ of the non-pregnant women of child-bearing age had malaria parasites. The prevalence of anaemia at old age was higher than young pregnant women. Among women aged $<20$ years, $6(60.0 \%)$ pregnant women and $17(58.6 \%)$ non-pregnant women of child-bearing age had anaemia. At age $<20$ years, there was a higher risk of anaemia $(\mathrm{OR}=1.27,95 \% \mathrm{CI}=0.6-2.7)$ among the non-pregnant women of child-bearing age than pregnant women $(\mathrm{OR}=$ $0.90,95 \% \mathrm{CI}=0.2-3.2$ ). The prevalence of anaemia in pregnant women was higher than in non-pregnant women of child-bearing age at age $\geq 20$ years. At age $\geq 20$ years, $232(62.7 \%)$ pregnant women and 185 (52.7\%) nonpregnant women were anaemic (Figure 2).

Malaria parasitaemia was found in $22(19.5 \%)$ nulliparous women and 26 (9.7\%) women who had one or more pregnancies with successful delivery. Compared to
Table 1. Prevalence of Plasmodium species in pregnant and non-pregnant women of child- bearing age.

\begin{tabular}{ccc}
\hline $\begin{array}{c}\text { Plasmodium } \\
\text { species }\end{array}$ & $\begin{array}{c}\text { Pregnant women } \\
\text { n (\%) }\end{array}$ & $\begin{array}{c}\text { Non-pregnant } \\
\text { women } \\
\text { n (\%) }\end{array}$ \\
\hline $\begin{array}{c}\text { Plasmodium } \\
\text { falciparum }\end{array}$ & $41(85.4 \%)$ & $19(76 \%)$ \\
$\begin{array}{c}\text { Plasmodium ovale } \\
\text { Plasmodium } \\
\text { malariae }\end{array}$ & $5(10.4 \%)$ & $4(16 \%)$ \\
\hline
\end{tabular}

Table 2. Prevalence of intestinal nematodes among the pregnant women.

\begin{tabular}{lcccc}
\hline & $\begin{array}{c}\text { Hb }< \\
\mathbf{1 1} \mathbf{g} / \mathbf{d l}\end{array}$ & $\begin{array}{c}\text { Anaemia } \\
\text { Prevalence } \\
\mathbf{n}(\mathbf{\%})\end{array}$ & $\begin{array}{c}\text { Odds Ratio } \\
\mathbf{( 9 5 \% ~ C I )}\end{array}$ & P-value \\
\hline \multicolumn{4}{c}{ Participants } \\
$380(100)$ & $238(62.6)$ \\
& Stool examination \\
Nematodes & $5(1.3)$ & $2(40.0)$ & $0.39(0.06478-2.380)$ & 0.3668 \\
$\begin{array}{c}\text { No } \\
\text { Nematode }\end{array}$ & $375(98.7)$ & $236(62.9)$ \\
\hline${ }^{*}=$ Reference category; CI $=$ Confidence interval; $\mathrm{n}=$ Number of subjects; \\
Hb = Haemoglobin level.
\end{tabular}

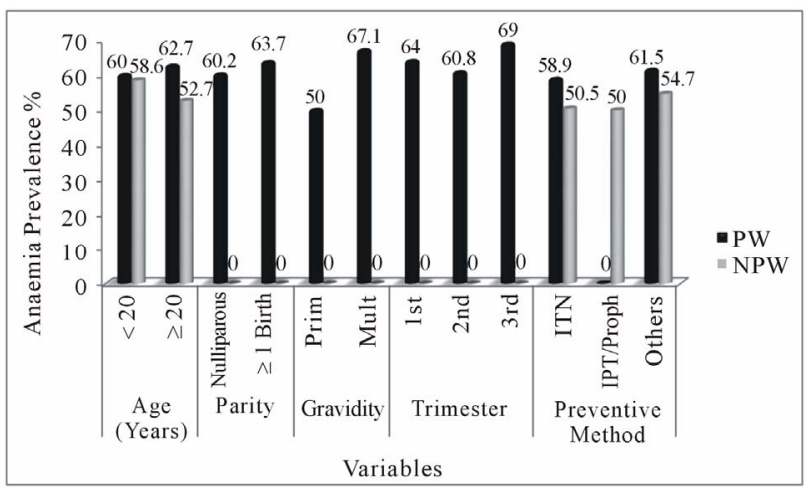

Figure 2. Comparison of anaemia in pregnant and nonpregnant women of child-bearing age. $\mathrm{PW}=$ Pregnant women, NPW = Non-pregnant women, Proph = Prophylaxis, $1^{\text {st }}=$ First trimester, $2^{\text {nd }}=$ Second trimester, $3^{\text {rd }}=$ Third trimester, Prim = Primigravidae, Multi $=$ Multigravidae, Pos $=$ Positive, Neg $=$ Negative.

multiparous women, there was a significantly higher risk of malaria parasitaemia in the nulliparous women $(\mathrm{OR}=$ 2.24) (Figure 3). Sixty eight (60.2\%) of the nulliparous women and $170(63.7 \%)$ multiparous women were anaemic. There was lower risk of anaemia in the nulliparous women $(\mathrm{OR}=0.86)$ using multiparity as reference category (Figure 2).

The pregnant women studied were divided into two gravidae; primigravidae and multigravidae. One hundred $(26.3 \%)$ were primigravidae while 280 (73.7\%) were 


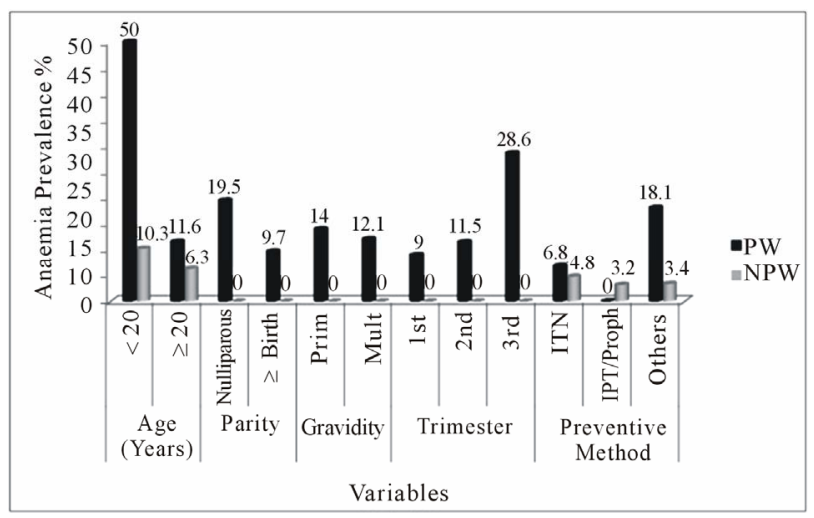

Figure 3. Comparison of malaria parasitaemia in pregnant and non-pregnant women of child-bearing age. $\mathbf{P W}=$ Pregnant women, NPW $=$ Non-pregnant women, Proph $=$ Prophylaxis, $1^{\text {st }}=$ First trimester, $2^{\text {nd }}=$ Second trimester, $3^{\text {rd }}=$ Third trimester, Prim $=$ Primigravidae, Multi $=$ Multigravidae.

multigravidae. The prevalence of malaria parasitaemia in multigravidae $(12.1 \%)$ was lower than in primigravidae $(14.0 \%)$, showing high risk $(\mathrm{OR}=1.18,95 \% \mathrm{CI}=0.6$ 2.3) (Figure 3). The prevalence of anaemia was higher in the multigravidae than in the primigravidae. Fifty $(50.0 \%)$ primigravidae and $188(67.1 \%)$ multigravidae were anaemic with lower risk in the primigravidae $(\mathrm{OR}=0.86$, $95 \% \mathrm{CI}=0.5$ - 1.4) (Figure 2).

The gestational period was divided into three stages; first trimester (0 - 12 weeks), second trimester (13 - 24 weeks) and third trimester (25 - 40 weeks). Among the pregnant women, $111(29.2 \%), 227(59.7 \%)$, and 42 $(11.1 \%)$ were in the first trimester, second trimester and third trimester respectively. The prevalence of malaria was $10(9.0 \%)$ in the first trimester, $26(11.5 \%)$ in the second trimester, and $12(28.6 \%)$ in the third trimester. Odds ratios of 0.25 at $95 \% \mathrm{CI}(0.10-0.63)$, and 0.32 at $95 \%$ CI $(0.15-0.71)$ were recorded in the first and second trimesters respectively with significant $\mathrm{P}$-values $(\mathrm{P}$-value $=0.0039$ for first trimester, $\mathrm{P}$-value $=0.0068$ for second trimester) (Figure 3).

The prevalence of anaemia was $71(64.0 \%), 138$ $(60.8 \%), 29(69.0 \%)$ in the first, second, and third trimesters respectively. Women in the third trimester had the highest anaemia prevalence $(69.0 \%)$ compared to $60.8 \%$ in the second trimester and $64.0 \%$ during the first trimester. The risk of anaemia was higher in the first trimester as compared to those in second trimester $(\mathrm{OR}=$ 0.80 at $95 \% \mathrm{CI}=0.38-1.70$ versus $\mathrm{OR}=0.70$ at $95 \% \mathrm{CI}$ $=0.34-1.41$ for first and second trimesters respectively) (Figure 2).

Preventive methods used by the pregnant women and non-pregnant women of child-bearing age included the use of Insecticide Treated Net (ITN), Prophylaxis and "others". The "others" comprised insecticide sprays, mosquito coils, mosquito repellents and creams. The prevalence of malaria in pregnant women showed that, 17 $(6.8 \%)$ were ITN users, $0(0.0 \%)$ were IPT users, and 40 (18.1\%) used "Others". There was a high risk in the use of "Others" $(\mathrm{OR}=4.17,95 \% \mathrm{CI}=1.90-9.19$, P-value $=$ 0.0001 ) as a preventive measure but low risk was associated with the use of ITN $(\mathrm{OR}=0.23,95 \% \mathrm{CI}=0.12$ 0.43 , P-value $<0.0001$ ) (Figure 3). Among the nonpregnant women of child-bearing age, malaria parasitaemia was found in $13(4.8 \%)$ ITN users, 3 (3.2\%) prophylaxis and $8(3.4 \%)$ "others" users. There was low risk of malaria parasitaemia in the non-pregnant women of child-bearing age in relation to preventive measures; (OR $=0.40,95 \% \mathrm{CI}=0.17-0.90$ for ITN, OR $=0.39,95 \%$ $\mathrm{CI}=0.11-1.33$ for prophylaxis, and $\mathrm{OR}=0.28,95 \% \mathrm{CI}$ $=0.12-0.66$ for "others") (Figure 2).

In the pregnant women, anaemia was recorded in 148 (58.9\%) ITN users, 0 (0.0\%) IPT users, and $136(61.5 \%)$ "others" users. Odds ratios of 0.62 at $95 \%$ CI (0.40 - 0.98) for ITN usage, and 0.89 at 95\% CI (0.59 - 1.36) for "others" was deduced from the analysis. There was an indication of low risk of anaemia associated with preventive methods (Odds ratios $<1$ ). Comparing this with nonpregnant women of child-bearing age revealed that, 138 (50.5\%) ITN users, and 127 (54.7\%) who used other means of control were anaemic. There was no significant association between anaemia and the use of "others" (OR $=1.18,95 \% \mathrm{CI}=0.78-1.78)$ whilst low risk was associated with the use of ITN $(\mathrm{OR}=0.69,95 \% \mathrm{CI}=0.44$ 1.08 ) in the non-pregnant women (Figure 2).

\section{Discussion}

Malaria parasitaemia and anaemia differed between age groups. Most of the respondents studied were old, suggesting low level of teenage pregnancy in the studied communities. This might be due to education and creating awareness concerning teenage pregnancy. In general, parasite density was higher in the young than the old women. This agreed with a study in Gabon with younger women more at risk [19]. This may be due to host or environmental factors [19]. It may also be attributed to age related immunity as a result of previous exposure to malaria in child-bearing years [19]. The high anaemia prevalence observed in pregnant women contradicts a study in Ghana which indicated that lower prevalence of anaemia was significantly associated with pregnant women [20].

Malaria parasitaemia was higher in nulliparous women than multiparous women. Women with successive births are believed to have had exposure to a variety of strains of malaria parasites, therefore developing somehow efficient immunity against most strains of the parasites [21, 22]. Peripheral and placental parasitaemia have been 
reported to decrease with increasing parity among pregnant women [23]. The present study confirmed that, parity is a variable that affects malaria parasitaemia (P-value $<$ $0.05)$. Multiparous women had higher prevalence $(63.7 \%)$ of anaemia than the nulliparous $(60.2 \%)$. A study in Iran found similar results [24]. However, Glover-Amengor and his colleagues in their study re- ported lower prevalence of anaemia which was strongly associated with increasing parity [20]. Anaemia in pregnancy could be attributable to several factors including decrease of iron from tissue, malnutrition and insufficient iron consumption [24].

The higher prevalence observed in primigravidae is consistent with other reports [4,19,25-27]. Anti-adhesion antibodies against chondroitin sulphate A-binding parasites are associated with protection from maternal malaria, but these antibodies develop only over successive pregnancies, hence the susceptibility of primigravidae to malaria infection compared to multigravidae [28]. Some authors also believed that primigravid women have little or no immunity against the infecting strains of Plasmodium and hence suffer adverse complications [29]. The occurrence of relatively more anaemia in multigravidae than in primigravidae confirmed an earlier study conducted in Nigeria where anaemia was found less in primigravidae [1]. This may be due to repeated pregnancies at shorter intervals without allowing for replenishment of the iron stores [1].

Many of the respondents in this study registered for antenatal care in their second and third trimester. This seems to be a common practice in Africa [30]. In a study conducted in Zaire, most of the pregnant women attended antenatal clinic (ANC) for the first time in their sixth or seventh month of gestation and made three to four visits before delivery [30]. This finding also confirmed results of a similar study conducted in Nigeria [31]. This practice is detrimental as it does not allow for early detection and correction of pregnancy associated complications such as anaemia. It is therefore very important to educate women on the need to register early for antenatal care. The mean Haemoglobin $(\mathrm{Hb})$ for the pregnant women in the last trimester in the present study was relatively low $(9.4 \mathrm{~g} / \mathrm{dl})$ compared to an earlier study in Ghana with a mean $\mathrm{Hb}$ value of $10.7 \mathrm{~g} / \mathrm{dl}$ [32]. It has been reported that anaemia is strongly associated with pregnant [33]. It is possible that better nutrition or increased iron intake may have resulted in reduced anaemia. However, the present study had no information on the diet of participants to evaluate this hypothesis.

Insecticide Treated Nets reduce human-vector contact by physically excluding vector mosquitoes, killing them if they land on ITNs or repelling them, thereby driving them away. Distribution of ITNs through ANC can help, but this does not address the effects of malaria before the first ANC visit [34]. Generally, anaemia was found to be higher in pregnant women than in non-pregnant women of child-bearing age who used ITNs. This prevalence is low compared to a study in Tanzania where $72 \%$ pregnant ITN users were anaemic [35]. With a significant $\mathrm{P}$-value, ITN usage is effective in controlling anaemia during pregnancy. This is supported by other studies where ITNs effectively reduce the prevalence of anaemia during pregnancy [36,37]. Among non-pregnant women, a study in Nigeria showed lower anaemia prevalence [38] compared with the present study. The use of 'others' made a significant impact on malaria parasitaemia in both pregnant and non-pregnant women of child-bearing age. In Uganda, mosquito coils were rarely used in malaria prevention by pregnant women [39]. The reason could be due to the possible health hazards associated with it. Although recommended, none of the pregnant women in the present study received IPT at ANC. This contradicts an earlier study where $26.4 \%$ of the women did not receive an IPT during ANC and only $34.2 \%$ received two doses [40]. In order to reduce the burden of malaria in pregnancy, it may be essential to establish a system of supervised intermittent preventive treatment with a safe and effective anti-malarial so as to control or reduce malaria parasitaemia in pregnant women [41].

The current findings revealed lower prevalence of intestinal nematodes among pregnant women. An earlier study reported an overall prevalence of $25.7 \%$ intestinal nematodes in pregnant women [40]. In addition, a study in Kenya contradicts the present study where $73 \%$ pregnant women had intestinal nematodes [42]. Even though it is clear that there is significant association between anaemia and intestinal nematodes [43], the outcome of the current study however indicated otherwise.

The predominant species isolated in both pregnant and non-pregnant women of child-bearing age was $P$. falciparum. This is because, $P$. falciparum is the main species found in the tropical and subtropical regions of Africa [18]. Plasmodium falciparum is also the most widespread species, accounting for up to $80 \%$ of malaria cases worldwide [18]. The present study agreed with a study in Pakistan [44], indicating $76.75 \%$ P. falciparum infection during pregnancy. In India, 58\% P. falciparum infection was recorded in the non-pregnant women of child-bearing age [45].

In summary, prevalence of malaria and anaemia were higher in pregnant women compared with their nonpregnant counterparts. Nduka and friends reported higher malaria parasitaemia in pregnant women $(54 \%)$ compared with non-pregnant women of child-bearing age (33\%) [46]. In the present study, $12.6 \%$ prevalence of malaria parasitaemia in pregnant women was lower compared with 23\% reported in Mozambique [47] and $26.75 \%$ reported in Malawi [48]. The prevalence of ma- 
laria in the present study among pregnant women was much lower than $47.5 \%$ reported in Onitsha, Nigeria, $42 \%$ reported in Ghana [32], 57.5\% reported in Gabon [19] and $41 \%$ observed in Uyo, Nigeria [49]. The high rate of malaria prevalence observed in the present study could be due to the environmental conditions inherent in urban and peri-urban areas, which favour malaria parasite transmission [46]. It has been recognized that a temperature range of $20^{\circ} \mathrm{C}-30^{\circ} \mathrm{C}$ and relative humidity of $60 \%$ and above were suitable for malaria parasite transmission [50]. Delay in starting pre-natal care during pregnancy may also have contributed to this prevalence [46]. In addition, the transient depression of cell-mediated immunity in pregnancy that allows foetal allograft retention but also interferes with resistance to various infectious diseases such as malaria could be a contributory factor [51]. Anaemia prevalence was higher in pregnant women. This agreed with work done by Nduka and colleagues [46]. Similar results were found in other studies [52-54]. The prevalence rate of anaemia observed in the present study agreed with the mean percentage for Africa which is $61 \%[19,55,56]$. Whitfield [57] and van den Broek [58] reported anaemia in pregnancy as multifactorial and may be caused by other factors other than malaria parasitaemia $[57,58]$. Any strategy aimed at combating this important public health challenge should aim at addressing all the potential causal factors after their elucidation.

\section{Conclusion}

In the present study, malaria parasitaemia and anaemia were common medical conditions associated with pregnancy. Pregnant women were more susceptible to malaria and anaemia than non-pregnant women of childbearing age. Malaria was not the only cause of anaemia and the main species of Plasmodium isolated in the blood samples was Plasmodium falciparum. Most of the pregnant women reported at ANC during the second trimester. The absence of IPT during the time of the study might have resulted in the relatively high prevalence of malaria in the pregnant women.

\section{Recommendation}

Malaria and anaemia can be controlled effectively with administration of IPT, folic acid tablets including balanced diet during pregnancy. Community Health Education should be provided to positively influence the knowledge and attitudes of pregnant and non-pregnant women of child-bearing age to malaria and anaemia, including early antenatal registration. Assessment of blood transfusion cases and other causes of anaemia apart from malaria are strongly recommended.

\section{Acknowledgements}

We thank the pregnant and non-pregnant women of child bearing age whose participation made the study and findings possible. We also thank the Medical Director of the University Hospital for giving us permission to carry out our research. Our gratitude goes to the Ethical Review Committee of the School of Medical Sciences, Kwame Nkrumah University of Science and Technology, Kumasi.

\section{REFERENCES}

[1] E. U. Nwonwu, P. C. Ibekwe, J. I. Ugwu, H. C. Obarezi and O. C. Nwagbara, "Prevalence of Malaria Parasitaemia and Malaria Related Anaemia among Pregnant Women in Abakaliki, South East Nigeria," Nigerian Journal of Clinical Practice, Vol. 12, No. 2, 2009, pp. 182-186.

[2] L. O. Omo-Aghoja, E. Abe, P. Feyi-Waboso and F. E. Okonofua, "The Challenges of Diagnosis and Treatment of Malaria in Pregnancy in Low Resource Settings," Acta Obstetricia et Gynecologica Scandinavica, Vol. 87, No. 7, 2008, pp.693-696. doi:10.1080/00016340802136889

[3] S. Lindsay, J. Ansell, C. Selman, V. Cox, K .Hamilton and G. Walraven, "Effect of Pregnancy on Exposure to Malaria Mosquitoes," The Lancet, Vol. 355, No. 9219, 2000, pp. 1972-1975. doi:10.1016/S0140-6736(00)02334-5

[4] I. A. McGregor, "Epidemiology, Malaria and Pregnancy," American Journal of Tropical Medicine \& Hygiene, Vol. 33, No. 4, 1984, pp. 517-525.

[5] S. Ramsay, "Preventing Malaria in Pregnancy," Lancet Infectious Diseases, Vol. 3, No. 1, 2003, p. 4. doi:10.1016/S1473-3099(03)00502-4

[6] A. C. Granja, F. Machungo, A. Gomes, S. Bergstrom and B. Brabin, "Malaria-Related Maternal Mortality in Urban Mozambique," Annals of Tropical Medicine and Parasitology, Vol. 92. No. 3, 1998, pp. 257-263. doi:10.1080/00034989859816

[7] F. Nosten, S. J. Rogerson, J. B. Beeson, R. McGready, T. K. Mutabingwa and B. Brabin, "Malaria in Pregnancy and the Endemicity Spectrum: What Can We Learn?" Trends in Parasitology, Vol. 20, No. 9, 2004, pp. 425-432. doi:10.1016/j.pt.2004.06.007

[8] C. J. Whitty, S. Edmonds and T. K. Mutabingwa, "Malaria in pregnancy," An International Journal of Obstetrics and Gynaecology, Vol. 112, No. 9, 2005, pp. 1189-1195. doi:10.1111/j.1471-0528.2005.00714.x

[9] C. E. Shulman, W. J. Graham, H. Jilo, B. S. Lowe, L. New, J. Obiero, R. W. Snow and K. Marsh, "Malaria is an Important Cause of Anaemia in Primigravidae: Evidence from a District Hospital in Coastal Kenya," Transactions of the Royal Society of Tropical Medicine and Hygiene, Vol. 90, No. 5, 1996, pp. 535-539. doi:10.1016/S0035-9203(96)90312-0

[10] WHO, "The Prevalence of Anaemia in Women: A Tabulation of Available Information," 1992.

[11] WHO/UNICEF/UNU, "Iron Deficiency Anaemia; Assess- 
ment, Prevention and, Control," 2001.

[12] WHO, "Prevention and Management of Severe Anaemia in Pregnancy," Report of a Technical Working Group, 1994.

[13] N. van den Broek, "Anaemia in Pregnancy in Developing Countries," British Journal of Obstetrics and Gynaecology, Vol. 105, No. 4, 1998, pp. 385-390. doi:10.1111/j.1471-0528.1998.tb10120.x

[14] WHO/AFRO, "A Strategic Framework for Malaria Prevention and Control during Pregnancy in the African Region," 2004.

[15] H. L. Guyatt and R. W. Snow, "The Epidemiology and Burden of Plasmodium Falciparum-Related Anemia among Pregnant Women in Sub-Saharan Africa," American Journal of Tropical Medicine \& Hygiene, Vol. 64, No. 1, 2001, pp. 36-44.

[16] J. L. Gallup and J. D. Sachs, "The Economic Burden of Malaria," American Journal of Tropical Medicine \& Hygiene, Vol. 64, No. 1-2, 2001, pp. 85-96.

[17] GSS, "Ghana Statistical Service," 2002.

[18] M. Cheesbrough, "District Laboratory Pratice in Tropical Countries," Cambridge University Press, Cambridge, 2005. doi:10.1017/CBO9780511581304

[19] M. K. Bouyou-Akotet, D. E. Ionete-Collard, M. MabikaManfoumbi, E. Kendjo, P. B. Matsiegui, E. Mavoungou and M. Kombila, "Prevalence of Plasmodium Falciparum Infection in Pregnant Women in Gabon," Malaria Journal, Vol. 2, No. 1, 2003 , p. 18. doi:10.1186/1475-2875-2-18

[20] M. Glover-Amengor, W. B. Owusu and B Akanmori, "Determinants of Anaemia in Pregnancy in Sekyere West District, Ghana," Ghana Medical Journal, Vol. 39, No. 3, 2005, pp. 102-107.

[21] J. G. Beeson, S. J. Rogerson, B. M. Cooke, J. C. Reeder, W. Chai, A. M. Lawson, M. E. Molyneux and G. V. Brown, "Adhesion of Plasmodium Falciparum-Infected Erythrocytes to Hyaluronic Acid in Placental Malaria," Nature Medicine, Vol. 6, No. 1, 2000, pp. 86-90. doi:10.1038/71582

[22] M. Fried and P. E. Duffy, "Adherence of Plasmodium Falciparum to Chondroitin Sulfate A in the Human Placenta," Science, Vol. 272, No. 5267, 1996, pp. 15021504. doi:10.1126/science.272.5267.1502

[23] C. E. Shulman and E. K. Dorman, "Importance and Prevention of Malaria in Pregnancy," Transactions of the Royal Society of Tropical Medicine and Hygiene, Vol. 97, No. 1, 2003, pp. 30-35. doi:10.1016/S0035-9203(03)90012-5

[24] G. R. Veghari, A. R. Mansourian and A. J. Marjani, "The Comparison of the Anemia in Pregnant and Non-Pregnant Women in the Villages of the South-East of Caspin SeaGorgan-Iran," Journal of Medical Sciences, Vol. 7, No. 2, 2007, pp. 303-306. doi:10.3923/jms.2007.303.306

[25] B. Brabin, "An Assessment of Low Birthweight Risk in Primiparae as an Indicator of Malaria Control in Pregnancy," International Journal of Epidemiology, Vol. 20, No. 1, 1991, pp. 276-283. doi:10.1093/ije/20.1.276

[26] C. Menendez, "Malaria during Pregnancy: A Priority Area of Malaria Research and Control," Parasitology Today,
Vol. 11, No. 5, 1995, pp. 178-183. doi:10.1016/0169-4758(95)80151-0

[27] C. E. Shulman, E. K. Dorman and J. N. Bulmer, "Malaria as a Cause of Severe Anaemia in Pregnancy," The Lancet, Vol. 360, No. 9331, 2002, p. 494. doi:10.1016/S0140-6736(02)09662-9

[28] P. E. Duffy and M. Fried, "Malaria during Pregnancy: Parasites, Antibodies and Chondroitin Sulphate A," Biochemical Society Transaction, Vol. 27, No. 4, 1999, pp. 478-482.

[29] S. Beck, F. P. Mockenhaupt, U. Bienzle, T. A. Eggelte, W. N. Thompson and K. Stark, "Multiplicity of Plasmodium Falciparum Infection in Pregnancy," American Journal of Tropical Medicine and Hygiene, Vol. 65, No. 5, 2001, pp. 631-636.

[30] R. W. Steketee, J. G. Breman, K. M. Paluku, M. Moore, J. Roy and M. Ma-Disu, "Malaria Infection in Pregnant Women in Zaire: The Effects and the Potential for Intervention," Annals of Tropical Medicine and Parasitology, Vol. 82, No. 2, 1988, pp. 113-120.

[31] F. E. Okonofua and O. R. Abejide, "Prevalence of Malaria Parasitaemia in Pregnancy in Nigerian Women," Journal of Obstetrics \& Gynaecology, Vol. 16, No. 5, 1996, pp. 311-315. doi:10.3109/01443619609030034

[32] F. P. Mockenhaupt, B. Rong, M. Gunther, S. Beck, H. Till, E. Kohne, W. N. Thompson and U. Bienzle, "Anaemia in Pregnant Ghanaian Women: Importance of Malaria, Iron Deficiency, and Haemoglobinopathies," Transactions of the Royal Society of Tropical Medicine and Hygiene, Vol. 94, No. 5, 2000, pp. 477-483. doi:10.1016/S0035-9203(00)90057-9

[33] P. Ouma, M. J. Hamel, M. Parise, J. G. Ayisi, K. Otieno, P. A. Kager and L. Slutsker, "Malaria and Anaemia among Pregnant Women at First Antenatal Clinic Visit in Kisumu, Western Kenya," Tropical Medicine and International Health, Vol. 12, No. 12, 2007, pp. 1515-1523. doi:10.1111/j.1365-3156.2007.01960.x

[34] H. L. Guyatt, M. H. Gotink, S. A. Ochola and R. W. Snow, "Free Bednets to Pregnant Women through Antenatal Clinics in Kenya: A Cheap, Simple and Equitable Approach to Delivery," Tropical Medicine and International Health, Vol. 7, No. 5, 2002, pp. 409-420. doi:10.1046/j.1365-3156.2002.00879.x

[35] T. Marchant, J. A. Schellenberg, T. Edgar, R. Nathan, S. Abdulla, O. Mukasa, H. Mponda and C. Lengeler, "Socially Marketed Insecticide-Treated Nets Improve Malaria and Anaemia in Pregnancy in Southern Tanzania," Tropical Medicine and International Health, Vol. 7, No. 2, 2002, pp. 149-158. doi:10.1046/j.1365-3156.2002.00840.x

[36] C. Gamble, J. P. Ekwaru and F. O. ter Kuile, "Insecticide-Treated Nets for Preventing Malaria in Pregnancy," Cochrane Database of Systemic Reviews, Vol. 19, No. 2, 2006, Article ID: CD003755.

[37] F. O. ter Kuile, D. J. Terlouw and P. A. Phillips-Howard, "Reduction of Malaria during Pregnancy by PermethrinTreated Bed Nets in an Area of Intense Perennial Malaria Transmission in Western Kenya," American Journal of Tropical Medicine and Hygiene, Vol. 68, No. 4, 2003, pp. 50-60. 
[38] O. Erhabor, T. C. Adias and M. L. Hart, "Effects of Falciparum Malaria on the Indices of Anaemia among Pregnant Women in the Niger Delta of Nigeria," Journal of Clinical Medicine and Research, Vol. 2, No. 3, 2010, pp. 35-41.

[39] A. K. Mbonye, S. Neema and P. Magnussen, "Preventing Malaria in Pregnancy: A Study of Perceptions and Policy Implications in Mukono District, Uganda," Health Policy and Planning, Vol. 21, No. 1, 2006, pp. 17-26.

[40] N. J. Yatich, J. Yi and T. Agbenyega, "Malaria and Intestinal Helminth Co-Infection among Pregnant Women in Ghana: Prevalence and Risk Factors," American Journal of Tropical Medicine and Hygiene, Vol. 80, No. 1, 2009, pp. 896-901.

[41] C. E. Shulman, E. K. Dorman, F. Cutts, K. Kawuondo, J. N. Bulmer, N. Peshu and K. Marsh, "Intermittent Sulphadoxine-Pyrimethamine to Prevent Severe Anaemia Secondary to Malaria in Pregnancy: A Randomised Placebo-Controlled Trial," The Lancet, Vol. 353, No. 9153, 1999, pp. 632-636. doi:10.1016/S0140-6736(98)07318-8

[42] P. W. Geissler, R. J. Prince, M. Levene, C. Poda, S. E. Beckerleg, W. Mutemi and C. E. Shulman, "Perceptions of Soil-Eating and Anaemia among Pregnant Women on the Kenyan Coast," Social Science \& Medicine, Vol. 48, No. 8, 1999, pp. 1069-1079. doi:10.1016/S0277-9536(98)00409-2

[43] G. Vasanthi, A. B. Pawashe, H. Susie, T. Sujatha and L. Raman, "Iron Nutritional Status of Adolescent Girls from Rural Area and Urban Slum," Indian Pediatrics, Vol. 31, No. 2, 1994, pp. 127-132.

[44] N. Saba, A. Sultana and I. Mahsud, "Outcome and Complications of Malaria in Pregnancy," Gomal Journal of Medical Sciences, Vol. 6, No. 2, 2008, pp. 26-29.

[45] N. Singh, M. M. Shukla, R. Srivastava and V. P. Sharma, "Prevalence of Malaria among Pregnant and Non-Pregnant Women of District Jabalpur, Madhya Pradesh," Indian Journal of Malariology, Vol. 32, No. 1, 1995, pp. 613.

[46] F. O. Nduka, A. Egbu, C. Okafor and V. O. Nwaugo, "Prevalence of Malaria Parasites and Anaemia in Pregnant and Non-Pregnant Women in Aba and Okigwe Towns of Southeast Nigeria," Animal Research International, Vol. 3, No. 3 2006, pp. 508-512.

[47] F. Saute, C. Menendez, A. Mayor, J. Aponte, X. GomezOlive, M. Dgedge and P. Alonso, "Malaria in Pregnancy in Rural Mozambique: The Role of Parity, Submicroscopic and Multiple Plasmodium falciparum Infections,"
Tropical Medicine \& International Health, Vol. 7, No. 1, 2002, pp. 19-28. doi:10.1046/j.1365-3156.2002.00831.x

[48] S. J. Rogerson, N. R. van den Broek, E. Chaluluka, C. Qongwane, C. G. Mhango and M. E. Molyneux, "Malaria and Anemia in Antenatal Women in Blantyre, Malawi: A Twelve-Month Survey," American Journal of Tropical Medicine and Hygiene, Vol. 62, No. 3, 2000, pp. 335340.

[49] K. N. Opara, E. S. Ibanga, N. B. Wali and L. P. Usip, "Falciparum Malaria and Susceptibility to Genetic Markers of Pregnant Women in Uyo, Southeast Nigeria," $A b$ stract of the 28th Annual Conference of the Nigeria Society of Parasitology held at Imo State University Owerri, 2004, p. 76.

[50] WHO, "WHO Expert Committee on Malaria," Twentieth Report, 2000.

[51] E. N. Meeusen, R. J. Bischof and C. S. Lee, "Comparative T-Cell Responses during Pregnancy in Large Animals and Humans," American Journal of Reproductive Immunology, Vol. 46, No. 2, 2001, pp. 169-179. doi:10.1111/j.8755-8920.2001.460208.x

[52] P. Nestel, A. Mellara, J. Rosado and J. O. Mora, "Nutrition of Hondurian Mothers/Caretakers," Pan American Journal of Public Health, Vol. 5, No. 3, 1999, pp. 164 171. doi:10.1590/S1020-49891999000300005

[53] H. Sadeghipour, M. Farahani and E. Moghrabi, "Prevalence and Causes of Iron Deficiency Anaemia in Iranian Women of Reproductive Age," Journal of Medical Council of Islamic Republic of Iran, Vol. 2, 2001, p. 1377.

[54] R. D. Stevens, "Anaemia the Scourge of the Third World," Health Millions, Vol. 26, No. 2, 2000, pp. 21-23.

[55] L. S. Nair and A. S. Nair, "Effects of Malaria Infection on Pregnancy," Indian Journal of Malariology, Vol. 30, No. 4, 1993, pp. 207-214.

[56] N. R. van den Broek, S. J. Rogerson, C. G. Mhango, B. Kambala, S. A. White and M. E. Molyneux, "Anaemia in Pregnancy in Southern Malawi: Prevalence and Risk Factors," An International Journal of Obstetrics \& Gynaecology, Vol. 107, No. 4, 2000, pp. 445-451. doi:10.1111/j.1471-0528.2000.tb13260.x

[57] C. R. Whitfield, "Blood Disorders in Pregnancy: Dewhurst's Textbook of Obstetrics and Gynaecology for Postgraduates, Carlton, Australia," Blackwell Science, Vol. 5, 1995, pp. 228-229.

[58] N. van den Broek, "The Cytology of Anaemia in Pregnancy in West Africa," Tropical Doctor, Vol. 26, No. 1, 1996, pp. 5-7. 